\title{
GRPR antagonists for prostate cancer-prospects and caveats
}

Rafael Roesler and Gilberto Schwartsmann

In their Review, Mansi and colleagues provide an excellent overview of the role of the gastrin-releasing peptide receptor (GRPR) in the progression of prostate cancer and other urological cancers, and discuss the potential of GRPR as a therapeutic target (Mansi, R., Fleischmann, A., Mäcke, H. R. \& Reubi, J. C. Targeting GRPR in urological cancers-from basic research to clinical application. Nat. Rev. Urol. 10, 235-244; 2013). ${ }^{1}$ As acknowledged by the authors, the first human trial of a GRPR antagonist was performed at our institution (G. Schwartsmann was the principal investigator). ${ }^{2}$ Our phase I study enrolled 25 patients with diverse types of advanced solid malignant tumours, six of whom (24\%) had prostate cancer and three patients $(12 \%)$ had kidney cancer. Patients were given either one or two daily subcutaneous injections of the selective GRPR antagonist RC-3095 at doses up to $96 \mu \mathrm{g} / \mathrm{kg}$. Although no objective tumour responses to the treatment were observed, there were no significant toxic effects related to drug administration, and a phase II study is planned. In our opinion, it is worth pointing out some additional issues not addressed by Mansi et al. ${ }^{1}$ that might be relevant for the clinical development of GRPR antagonists for prostate cancer.

Regarding the cellular mechanisms involved in GRPR-driven tumour progression, prostate cancer might have a unique response to GRPR activation owing to a close functional interaction between the GRPR and the androgen receptor (AR). GRP-the endogenous GRPR agonist in humans, detected in $50-60 \%$ of prostate cancer specimens ${ }^{3}$ - can activate the AR and enhance the proliferation and migration of prostate cancer cells in androgendepleted conditions. Importantly, androgen-independent growth of prostate cancer cells transfected to overexpress GRP requires GRPR activation and is inhibited by RC-3095. ${ }^{4}$ These findings strongly indicate that the GRPR can mediate cancer initiation and growth by modulating AR signalling, even in the absence of androgen stimulation. Thus, the GRPR is a particularly promising target for the inhibition of tumour progression in patients with castration-resistant prostate cancer. ${ }^{4,5}$ Further investigation of the crosstalk between GRPR and AR signalling is crucial for the characterization of therapeutic opportunities to block the GRPR in prostate cancer.

Other aspects of GRPR involvement in prostate cancer might, however, represent caveats that should be taken into account when designing clinical studies. For example, recent evidence suggests that GRPR overexpression is found predominantly in prostate tumours of small size and low grade. GRPR expression was inversely correlated with both Gleason score and preoperative PSA level..$^{6}$ These findings suggest that the clinical use of GRPR antagonists in patients with advanced disease might be limited.

Finally, although the antitumour effects of GRPR blockade have been demonstrated in prostate cancer animal models, these experiments have focused on evaluating the effects of GRPR antagonist monotherapy. Given the biological complexity of prostate cancer, targeting a single growth factor receptor or signalling pathway is not likely to have a substantial effect on prostate cancer growth. In a preclinical study using a rat model of glioma, we showed that although treatment with RC-3095 significantly reduced tumour growth, optimal response (and, in some animals, virtually complete tumour remission) was observed only when the GRPR antagonist was combined with the standard chemotherapeutic agent temozolomide. ${ }^{7}$ Preclinical studies in prostate cancer should, therefore, be designed with the purpose of characterizing the effects of GRPR antagonists when combined with other molecularly targeted agents or cytotoxic chemotherapy regimens.

Department of Pharmacology, Institute for Basic Health Sciences (R. Roesler), Department of Internal Medicine, School of Medicine (G. Schwartsmann),

Federal University of Rio Grande do Sul, Rua Ramiro Barcelos 2350,

90035-003 Porto Alegre RS, Brazil.

Correspondence to: R. Roesler

rafael.roesler@pq.cnpq.br

Acknowledgements

The authors are supported by the National Council for Scientific and Technological Development (CNPq; grant numbers 303703/2009-1 and $484185 / 2012-8$ to R. R.); the National Institute for Translational Medicine (INCT-TM); the University Hospital Research Fund (FIPE-HCPA); and the South American Office for Anticancer Drug Development.

Competing interests

The authors declare no competing interests.

1. Mansi, R., Fleischmann, A., Mäcke. H. R. \& Reubi, J. C. Targeting GRPR in urological cancers-from basic research to clinical application. Nat. Rev. Urol. 10, 235-244 (2013).

2. Schwartsmann, G. et al. A phase I trial of the bombesin/gastrin-releasing peptide (BN/GRP) antagonist RC-3095 in patients with advanced solid malignancies. Invest. New Drugs 24, 403-412 (2006).

3. Constantinides, C. et al. Immunohistochemical detection of gastrin releasing peptide in patients with prostate cancer. World J. Urol. 21, 183-187 (2003).

4. Yang, J. C. et al. Aberrant activation of androgen receptor in a new neuropeptideautocrine model of androgen-insensitive prostate cancer. Cancer Res. 69, 151-160 (2009).

5. Amorino, G. P. \& Parsons, S. J. Neuroendocrine cells in prostate cancer. Crit. Rev. Eukaryot. Gene Expr. 14, 287-300 (2004).

6. Beer, M. et al. Profiling gastrin-releasing peptide receptor in prostate tissues: clinical implications and molecular correlates. Prostate 72, 318-325 (2012).

7. de Oliveira, M. S. et al. Anti-proliferative effect of the gastrin-release peptide receptor antagonist RC-3095 plus temozolomide in experimental glioblastoma models. J. Neurooncol. 93, 191-201 (2009). 